M.I. Baranov

\title{
AN ANTHOLOGY OF THE DISTINGUISHED ACHIEVEMENTS IN SCIENCE AND TECHNIQUE. PART 53: NOBEL PRIZE LAUREATES IN PHYSICS FOR 2016-2019
}

Purpose. Brief presentation of the distinguished achievements of Nobel Prize Laureates in physics for current time period of 2016-2019. Methodology. Scientific methods of collection, analysis and analytical treatment of scientific and technical information of world level in area of astronomy, astrophysics, physicists of the condensed environments, laser physics, modern theoretical and experimental physics. Results. The brief analytical review of the scientific discovery and distinguished achievements of scientists-physicists in area of modern physical and technical problems which were marked the Nobel Prizes in physics for the period 2016-2019 is presented. In the number of such discovery and achievements of the best representatives of world scientific association entered: theoretical discovery of topology phase transitions and topology phases of matter, experimental discovery of gravity waves, revolutionary inventions in area of laser physics, astronomic discovery and contribution to our understanding of evolution of Universe and place of Earth in space. Originality. Systematization and analytical exposition is executed in short concentrated and accessible to the wide reader to the form of the known scientific and technical materials, awarded with the Nobel Prizes in physics for current time period of 2016-2019 and devoted to: the theoretical openings of topology phase transitions and topology phases of matter, experimental opening of gravity waves, revolutionary inventions in area of laser physics, astronomic discovery and contribution to our understanding of evolution of Universe and place of Earth in space. Practical value. Popularization and deepening of scientific and technical knowledge for students, engineer and technical specialists and research workers in area of astronomy, astrophysics, physicists of the condensed environments, laser physics, modern theoretical and experimental physics, extending their scientific range of interests and further development of scientific and technical progress in human society. References 22, figures 14.

Key words: Nobel prize on physics, distinguished scientific and technical achievements, theoretical discovery of topology phase transitions and topology phases of matter, experimental discovery of gravity waves, revolutionary inventions in area of laser physics, astronomic discovery and contribution to our understanding of evolution of Universe and place of Earth in space.

Наведено короткий аналітичний огляд видатних науково-технічних досягнень вчених, які відмічені Нобелівською премією по фізиці за період 2016-2019 рр. У число таких досягнень представників світової наукової спільноти увійшли: теоретичні відкиття топологічних фазових переходів і топологічних фаз матерії, експериментальне відкриття гравітаційних хвиль, революційні винаходи в галузі лазерної фізики, астрономічні відкриття і внесок в наше розуміння еволюції Всесвіму і місця Землі в космосі. Бібл. 22, рис. 14.

Ключові слова: Нобелівська премія по фізиці, видатні науково-технічні досягнення, теоретичні відкриття топологічних фазових переходів і топологічних фаз матерії, експериментальне відкриття гравітаційних хвиль, революційні винаходи в галузі лазерної фізики, астрономічні відкриття і внесок в наше розуміння еволюції Всесвіту i місця Землі в космосі.

Приведен краткий аналитический обзор выдающихся научно-технических достижений ученых, отмеченных Нобелевской премией по физике за период 2016-2019 г2. В число таких достижений представителей мирового научного сообщества вошли: теоретические открытия топологических фазовых переходов и топологических фаз материи, экспериментальное открытие гравитационных волн, революционные изобретения в области лазерной физики, астрономические открытия и вклад в наше понимание эволюции Вселенной и места Земли в космосе. Библ. 22, рис. 14.

Ключевые слова: Нобелевская премия по физике, выдающиеся научно-технические достижения, теоретические открытия топологических фазовых переходов и топологических фаз материи, экспериментальное открытие гравитационных волн, революционные изобретения в области лазерной физики, астрономические открытия и вклад в наше понимание эволюции Вселенной и места Земли в космосе.

Introduction. As it is well known, the Nobel Prize in physics is awarded once a year by the Royal Swedish Academy of Sciences. This is one of the five Nobel Prizes (in the field of physics, chemistry, physiology, literature and the establishment of peace between peoples), which appeared in the scientific world by will (this fact was documented in 1895 [1]) by the famous Swedish engineer and inventor of chemical explosives Alfred Nobel (18331896), which has been awarded since 1901 to living physicists for their outstanding scientific and technical achievements [2] «tested» by time [2]. We point out that in monetary terms, this prestigious international award for its more than a hundred years period underwent changes due to the financial condition of the Nobel Foundation and in 2017 amounted to 9 million Swedish kronor (about USD 1.12 million) [3]. The official ceremony of presenting this valuable award according to the established tradition takes place on December 10 of each year (on the day of A. Nobel's death) in the Swedish capital of Stockholm and the King of Sweden is solemnly awarded the prize to its laureates with no more than three people (the Nobel Prize on peace is an exception to this tradition, the ceremony of delivery of which is held 
annually in the capital of Norway the city of Oslo at the above time).

Earlier, the author in [2, 4-8] described the main outstanding achievements in science and technology of Nobel Prize winners in physics for the modern time period 1990-2015. We try below to present briefly similar scientific and technological achievements of world physicists in recent years, limited by the time period of 2016-2019.

The goal of the paper is a summary of the outstanding achievements in science and technology of Nobel Prize winners in physics for the modern time period, covering 2016-2019.

1. Nobel Prize in physics for 2016. The Laureates of this prestigious prize for 2016 were American-British theoretical physicists John Michael Kosterlitz (born on June 22, 1943, Fig. 1) and David James Thouless (born on September 21, 1934, Fig. 2) and Frederick Duncan Michael Haldane (born on September 14, 1951, Fig. 3), working in the USA [1]. The Nobel Prize to these physicists was not awarded for one specific scientific discovery, but for a whole list of pioneering scientific works that during the period 1970-1980 stimulated the development of a new direction in condensed matter physics [1]. It should be noted that the corresponding decision of the Nobel Committee it is indicated that this Prize was awarded to M. Kosterlitz, D. Thouless and D. Haldane «... for the theoretical discoveries of topological phase transitions and topological phases of matter» [1].

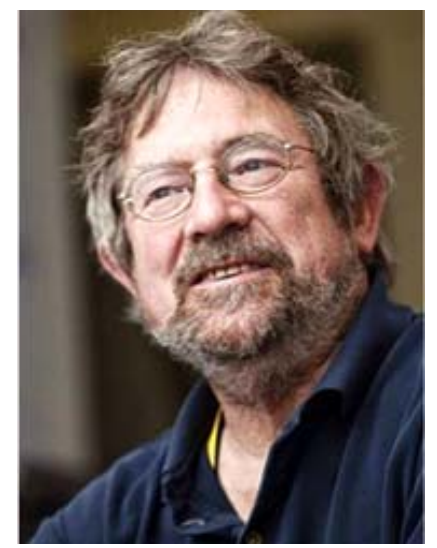

Fig. 1. Outstanding American-British theoretical physicist John Michael Kosterlitz (born in 1943), Laureate of Nobel Prize in Physics for 2016 [1]

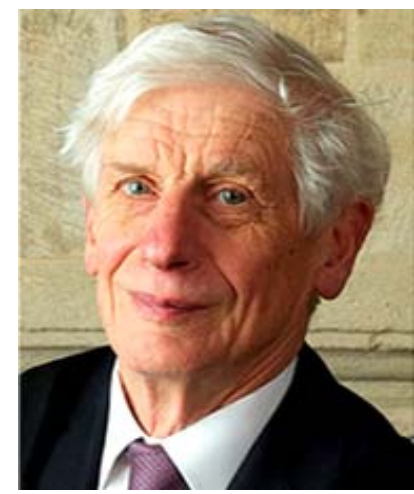

Fig. 2. Outstanding American-British theoretical physicist David James Thouless (born in 1934), Laureate of Nobel Prize in Physics for 2016 [1]

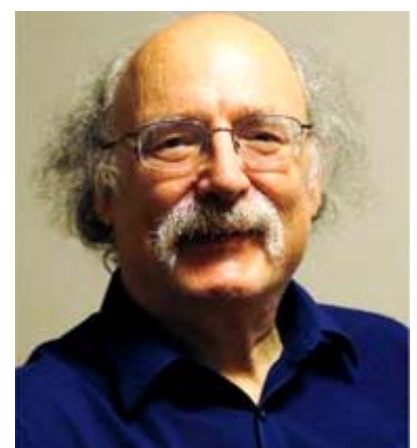

Fig. 3. Outstanding American-British theoretical physicist Frederick Duncan Michael Haldane (born in 1951), Laureate of Nobel Prize in Physics for 2016 [1]

In earthly nature, it so happened that condensed matter physics turned out to be the richest section of physics for various phenomena. For clarity of the complex scientific material presented below, we 1 answer, first of all, the simple question: what is understood in physics as a condensed medium? A condensed medium is a set of particles of the same type of substance that come together and strongly affect each other [9]. To condense (comes from the Latin word «condensare» - «to condense» [10]) a substance means to turn it from a gaseous state into a liquid or solid crystalline one. Therefore, a rarefied gas is not a condensed medium. In a condensed medium, a wealth of physical phenomena arises spontaneously dynamically as a result of collective effects [1]. In this regard, the properties of a condensed medium are determined by the synchronous collective movement of its enormous number of particles, and not its individual particles. It turns out that in the medium under consideration of these collective movements there is a huge variety of these of these collective movements.

In the works of the foreign theoretical physicists mentioned above, it was found that a condensed medium can «speak» not only the «language» of synchronous collective movements of its particles, but also the «language» of topologically nontrivial excitations [1]. For the reader, this physical concept requires its explanation. The term «topology» comes from the Greek words «topos» - «place» and «logos» - «word» [10]. In this regard, topology, as a branch of mathematics, studies the general properties of geometric figures that do not change during any continuous transformations of these figures [10]. It follows from such a branch of mathematics as geometry that if one geometric figure can be reduced by smooth deformation to another, then such two figures are considered topologically equivalent. If two geometric figures are not transformed into each other by any smooth changes, then such figures are considered topologically different. Next, we dwell on the term «excitation». In condensed matter physics, excitation is any collective deviation from the «dead» stationary state of the medium and its particles, that is, from the state with the least energy [1]. The oscillating excitation of the crystalline structure of a substance, characterized by the presence of sound waves (phonons) in it, can be caused by both external causes (e.g., mechanical shock to the crystal) and 
internal spontaneous causes (e.g., the presence of a nonzero temperature in the crystal). The usual thermal tremor of the crystal lattice of matter, which is widely known to us from the course of atomic physics, is due to the mutual overlapping of oscillating excitations (phonons) with different wavelengths [9]. In the case when the concentration of phonons in the crystal is high, a phase transition occurs in it and the crystal melts. From this we can conclude that as soon as theoretical physicists begin to understand in terms of what excitations one or another condensed medium should be described, they get the scientific «key» to its thermodynamic and other physical properties in their hands.

It is known that by controlling the amplitude of sound oscillations (phonons), one can smoothly change the strength (energy) of these vibrations: from their maximum to their minimum (zero). Therefore, phonons are topologically trivial excitations of matter. Just as the world around us is diverse, so are its condensed media. In some such media, there are excitations that cannot be smoothly reduced to zero. Moreover, it is not physically impossible, but fundamentally impossible (their form does not allow). In them, the excitations in their form are topologically different from the above phonons. In this case, there is no smooth operation that would translate a physical system (medium) with excitation into a similar system with the lowest energy. The properties of such physical media change stepwise. It was topology that allowed physicists to describe the properties of matter, which change stepwise.

A group of American theoretical physicists composed of M. Kosterlitz, D. Thouless and D. Haldane, who are British by origin, using topological methods of mathematics as a scientific tool, succeeded in the course of their many years of theoretical research in the period 1970-1980 to obtain a mathematical description of stepwise changing parameters in condensed matter [1]. They showed that in condensed systems such as twodimensional superconductors, phase transitions are very specific and are really associated with topological features (excitations) - vortices that are generated by «vortex antivortex» pairs [1]. These physicists have developed advanced mathematical methods to describe the unusual phases and properties of matter. For example, for such widely known materials as superconductors, superfluid liquids, and magnetic films. For objectivity in this complex and important scientific issue, it should be noted that in 1971 the Soviet theoretical physicist Vadim Berezinsky (1935-1980), who worked at the L.D. Landau Institute of Theoretical Physics of the Academy of Sciences of the USSE, for the first time in the world, put forward the idea that phase transitions in thin layers of superconductors and superfluid (quantum) liquids are based on topological excitations - vortices that spontaneously arise in matter in the form of coupled pairs «vortex - antivortex» (by the way, this a scientific idea formed the basis of his $\mathrm{PhD}$ Thesis) [1]. At the finite temperature of the substance, such pairs can be produced during a smooth local intrastructural change. It is they (these vortex pairs) that destroy in the two-dimensional case the crystalline phase of matter at low temperatures. Therefore, it is not for nothing that this phase transition in a condensed medium began to be called the «BerezinskyKosterlitz-Thouless» phase transition [11]. In this regard, in the Nobel Prize considered there is also a certain Russian «trace». We also point out that the most important works of the Soviet physicist V. Berezinsky relate to the theory of phase transitions in twodimensional systems and the theory of localization in disordered one-dimensional conductors. It was he who first theoretically showed that a thin (of the order of several Angstroms) film of liquid helium at low temperatures has the property of superfluidity [1].

In 1972, M. Kosterlitz and D. Thouless (a year later than our V. Berezinsky), realizing the important role of topological excitations, came to a similar conclusion about the determining effect of coupled «vortex antivortex» pairs in phase transitions in matter [11]. They went further and found that as the temperature of a substance increases, so many coupled «vortex antivortex» pairs accumulate in it that individual pairs become untwisted. In this case, in a condensed medium, the physical picture changes dramatically and its thermodynamic characteristics undergo stepwise changes: a phase transition occurs in the medium due to unraveling of topological excitations [1]. In a press release of the Nobel Committee in connection with the award of the indicated group of physicists of the corresponding Prize for 2016, it was said [12]: «... Scientists have opened the door to an unknown world where matter can take on «strange states». They used advanced mathematical methods to study unusual phases or states of matter. The work of scientists can be further used in science and electronics». They refuted the theories of superconductivity and superfluidity of matter that existed at that time, which argued that these phenomena can only occur in thin layers of matter. These physicists theoretically showed that in a substance the phenomenon of superconductivity can occur exclusively at low temperatures. They gave an accurate explanation of this physical mechanism based on phase transitions and found that this phenomenon disappears due to these transitions at higher temperatures [11]. Note that today the topological description of phase transitions in matter is used not only in the case of a thin layer, but also for ordinary three-dimensional materials. Further development of this field of physics led to the creation and study of a new class of substances - topological insulators that are popular today [11]. Over the past decade, this area of physics has been expanded by numerous studies aimed at finding unusual phases of matter. Now many researchers around the world are creating new topological materials that can be used in superconductors, new-generation electronics, and quantum computers $[1,11]$.

2. Nobel Prize in physics for 2017. The 2017 Nobel Laureates in Physics were distinguished experimental physicists who worked fruitfully in the United States, 
Rainer Weiss (born on September 29, 1932, Fig. 4), Barry Clark Barish (born on January 27, 1936, Fig. 5) and Kip Stephen Thorne (born on June 1, 1940, Fig. 6) «... for the decisive contribution to the creation of the LIGO detector and the registration of gravitational waves» [13].

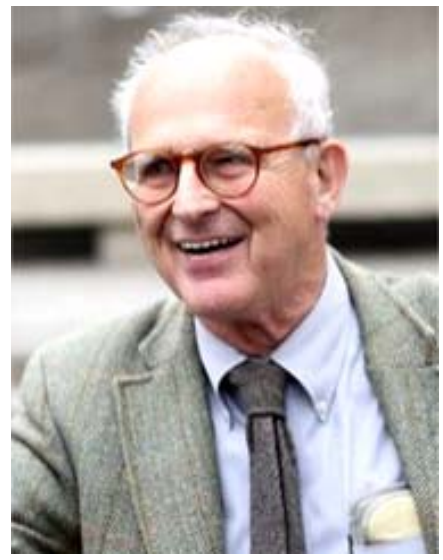

Fig. 4. Outstanding American experimental physicist Rainer Weiss (born in 1932), Laureate of Nobel Prize in Physics for 2017 [13]

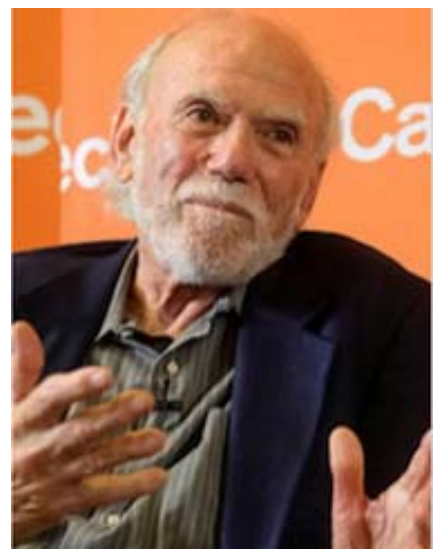

Fig. 5. Outstanding American experimental physicist Barry Clark Barish (born in 1936), Laureate of Nobel Prize in Physics for 2017 [13]

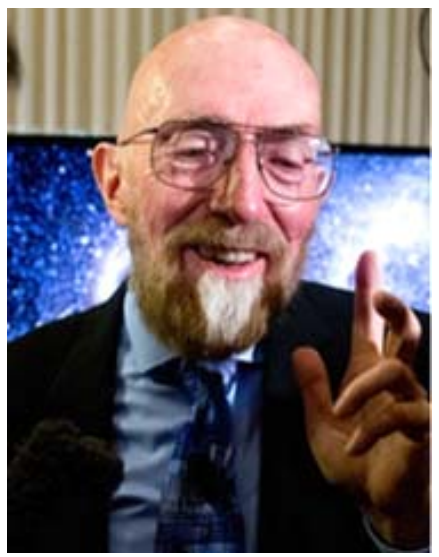

Fig. 6. Outstanding American experimental physicist Kip Stephen Thorne (born in 1940), Laureate of Nobel Prize in Physics for 2017 [13]

American scientists R. Weiss (Professor of physics at the Massachusetts Institute of Technology), as well as B. Barish and K. Thorne (Professor of physics at the
California Institute of Technology), who worked in the well-known scientific collaboration LIGO (Laser Interferometric Gravitational Observatory), on September 14, 2015 for the first time the world, using laser interferometers, discovered cosmic perturbations of «space-time» (gravitational waves) from the merger of a pair of «black holes» in our Universe [14].

To date, four signals (gravitational waves) have been recorded in observatories of the world from the confluence of «black holes» in the outer space surrounding planet Earth or the explosion of other massive objects $[14,15]$. The last discovery of LIGO (USA) was made in conjunction with the European observatory VIRGO. The existence of gravitational waves is one of the predictions of the general theory of relativity (GTR). Their discovery confirms not only GTR itself, but is also considered one of the proofs of the existence of «black holes» in space [14].

The above complex physical concepts require their explanation. To begin with, we point out that gravitational waves are oscillations of the space-time geometric structure propagating in "space-time» that move at the speed of light [14]. Their existence was predicted more than a hundred years ago in GTR, developed in 1915 by the outstanding German theoretical physicist Albert Einstein (1879-1955 [16]). To this should be added that the idea that the curvature of space can move in the form of a wave was first expressed by the famous English mathematician William Clifford (1845-1879) in 1876, i.e. almost 39 years before the creation by A. Einstein of GTR [14]. An interesting circumstance is that at the beginning of the 20th century, after A. Einstein developed the special theory of relativity (STR) in 1905, some wellknown scientists (including the prominent French mathematician and mechanic Henri Poincaré, 1854-1912 [16]) tried to include also gravity to STR. Note that A. Poincaré at one time interpreted gravity waves as waves moving in space similarly to electromagnetic waves [17]. However, only in 1915, A. Einstein in the framework of GTR was able to give a generally covariant description of gravity as a geometric effect. In such a way actually the modern theory of gravity was born. Nevertheless, the situation with the physical reality of gravitational waves in the scientific world still remained ambiguous. Here, scientific disputes revolved around the fundamental question: do gravitational waves even transfer energy or not? If they transfer it, then physicists have a chance to study them experimentally. If not, then they (gravitational waves) become a «thing in themselves» that is inaccessible to us for a physical experiment. For many years, a convincing answer to the above question in physical science did not exist. The final confirmation of the tangibility of gravitational waves came to modern physics only in the 1970s, when it became clear that a cosmic pulsar of the PSR B1913+16 type loses its energy due to gravitational radiation exactly as A. Einstein's GTR predicts by this physical phenomenon [17].

The LIGO laser-interferometric gravitational-wave observatory (USA), which now employs more than a 
thousand employees, recorded a gravitational wave for the second time in February 2016, which came to us from vast space [18]. The Nobel Committee would not award a Prize for only one such event, set by the LIGO detector in September 2015 [14]. When this gravitational wave (oscillations of «space-time») reached planet Earth, it was barely perceptible. In order to «reach» the Earth, this wave took more than a billion years [18]. The energy of the gravitational wave at its origin is huge, but its amplitude is incredibly small. To register it, physicists needed fundamentally new supersensitive instruments detectors of gravitational waves diverging in space from their origin at the speed of light in all directions. The place of their origin is, for example, distant cosmic places where very massive objects collide with each other «black holes» $[14,15]$. Far, far beyond the boundaries of our Milky Way galaxy, two «black holes» once crashed into each other and after 1.3 billion years the LIGO gravitational-wave observatory, located in the United States (Fig. 7), recorded this space event. The creation of these gravitational wave detectors required the work of thousands of people and the resources of 20 countries, first implemented in the USA in LIGO laser interferometers. To evaluate the scientific problems facing foreign experimental physicists and compare them with earthly problems that we understand, we note that in order to register a gravitational wave, scientists needed to achieve a sensitivity of their laser interferometers that was equivalent to the sensitivity of an instrument capable of measuring the distance to a distant star accurate to tenths of a millimeter [18].

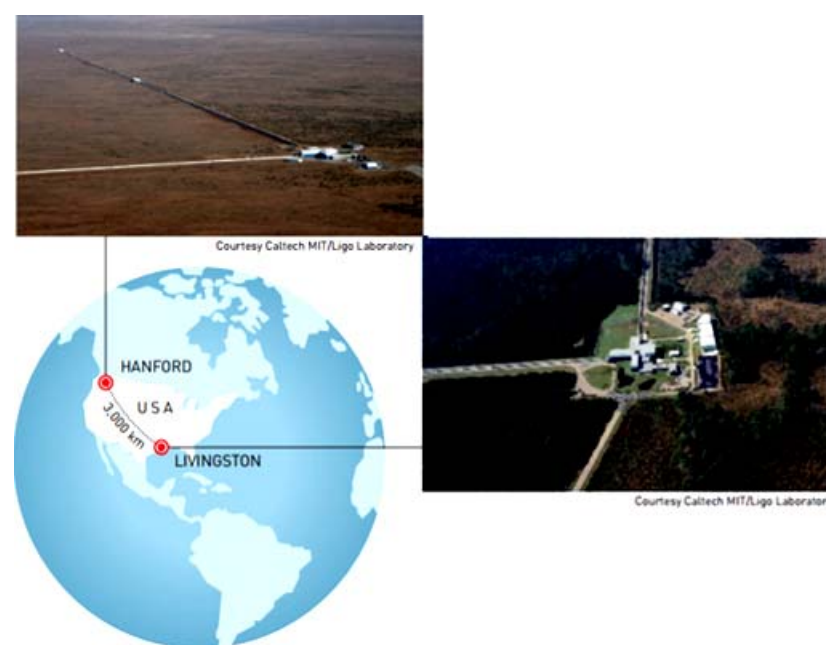

Fig. 7. Layout and general views of two American LIGO gravitational observatories in Livingston (Louisiana, USA) and in Hanford (Washington, USA) located at a distance of $3002 \mathrm{~km}$ from each other [18]

The reader may have simple questions: why do we even need earthlings and why spend huge sums of money on recording space disasters of the distant past? The answer may be adequate: for people to understand the world in which humanity exists. In the $1970 \mathrm{~s}, \mathrm{R}$. Weiss developed the concept of creating the LIGO detector in the USA, which would include two gravitational-wave observatories that are distant from each other at a distance of about $3002 \mathrm{~km}$ [18]. In it, he took into account those earthly phenomena that can distort the process of registration of a gravitational wave. In addition, he suggested ways to eliminate them and influence on the process of measuring this very weak wave amplitude. In a LIGO detector, a gravitational wave travels the distance between its two laser interferometers in $7 \mathrm{~ms}$. Therefore, in this huge supersensitive device, two laser interferometers can refine each other's readings. B. Barish joined the LIGO team, with only 40 employees at that time, in 1994 and turned it over time into the huge international collaboration LIGO-VIRGO (the last one is the recently created European gravitational-wave observatory). Thanks to the coordinated work of the participants of this scientific collaboration, a fundamental breakthrough in the physical registration of gravitational waves and understanding of gravity became possible [18]. $\mathrm{K}$. Thorne, as a leading world expert in the field of the theory of relativity, performed the theoretical calculations necessary for the operation of the LIGO detector [18]. It is interesting to note that the fourth gravitational wave recently detected by the LIGO detector was not the result of the merging of «black holes» in space, but the result of the explosion of a neutron star [18]. The studies conducted by R. Weiss, B. Barish, and K. Thorne expanded our knowledge about the Universe and led to the development of fundamentally new observation channels for distant space objects that develop multichannel astronomy, including gravitational wave astronomy [13].

3. Nobel Prize in physics for 2018. On October 2, 2018, the Royal Swedish Academy of Sciences announced the awarding of the next Nobel Prize in physics. Its winners «... for revolutionary inventions in the field of laser physics» became [19]: American physicist Arthur Ashkin (born on September 2, 1922, Fig. 8), American-French physicist Gérard Albert Mourou (born on June 22, 1944, Fig. 9) and Canadian physicist Donna Strickland (born on May 29, 1959, Fig. 10). This time, the Nobel Committee press release emphasized that D. Strickland became the third woman in history to be the winner of this prestigious physics Prize (after French-Polish radiochemist Maria SkłodowskaCurie (1867-1934) and American-German physicist Maria Göppert-Mayer (1906-1972) [16]).

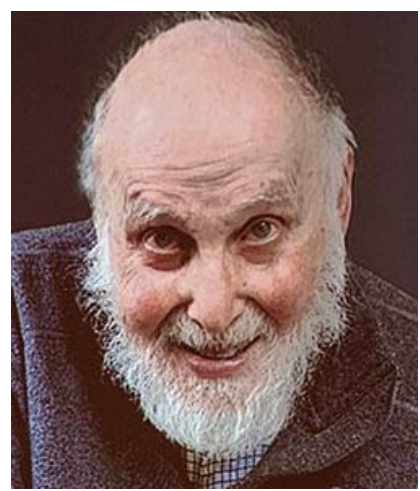

Fig. 8. Outstanding American physicist Arthur Ashkin (born in 1922), Laureate of Nobel Prize in Physics for 2018 [19] 


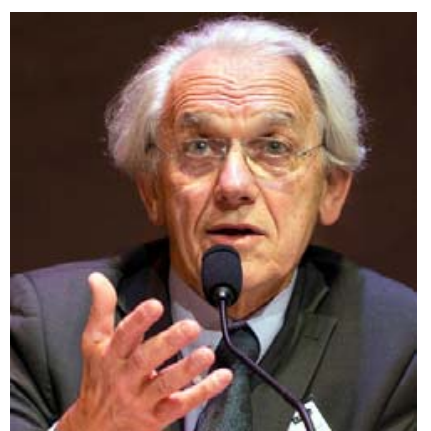

Fig. 9. Outstanding American-French physicist Gérard Albert Mourou (born in 1944), Laureate of Nobel Prize in Physics for 2018 [19]

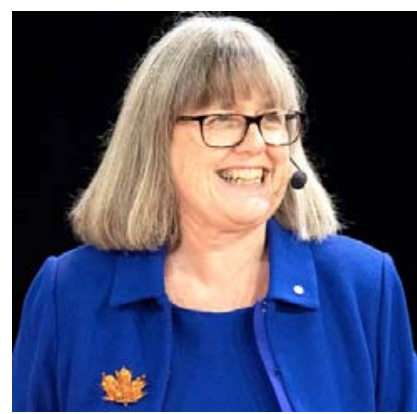

Fig. 10. Outstanding Canadian physicist Donna Strickland (born in 1959), Laureate of Nobel Prize in Physics for 2018 [19]

The winners of the 2018 Prize, named after A. Nobel, were awarded for their scientific work in the field of physical optics and laser technology, which they performed dozens of years ago. An interesting fact is that A. Ashkin was the oldest Nobel Prize winner in its entire history [19]. By the way, this scientist until 1992 headed the Department of Physical Optics and Electronics at Bell Labs, USA. Parents of A. Ashkina before the 1917 revolution in the Russian Empire left Odessa city for the United States. Remarkable inventions in the field of laser physics by A. Ashkin, G. Mourou and D. Strickland greatly expanded the practical application of light pressure (photons) in the world, which was made possible thanks to the tremendous progress of quantum optical generators - lasers («Light Amplification by Stimulated Emission of Radiation» [16]. Just from the official formulation of the Nobel Committee for the scientific merits of the above new physicist Laureates their applied orientation with obvious technological application follows. The last time a similar event in the history of the «Nobel» was observed in 2014, when three Japanese scientists were awarded the Nobel Prize in physics for the outstanding invention of blue LEDs $[8,19]$.

Note that in the period 2015-2017 the Nobel Prizes in physics (see Sections 1, 2 of this paper and [8]) were awarded to scientists for their outstanding results in the field of basic research. It is known that the pressure of the photon flux (ordinary light) is extremely small [9]. For example, the force with which sunlight pushes planet Earth in outer space is sixty trillion times less than the force of its attraction by the Sun [19]. Therefore, it was no coincidence that in 1905 the famous English physicist John Henry Poynting (1876-1943) in his presidential address to the British Physical Society noted that experiments performed in the world (including Professor at Moscow University Peter Nikolaevich Lebedev, 18661912) to determine the value of light pressure demonstrated the extreme smallness of this physical effect, «... excluding it from consideration in earthly affairs» [19]. This conclusion in physical science remained almost right up to the invention of lasers in quantum electronics $[9,16]$.

It is known that laser radiation has such rare physical properties as: exceptional spectral purity (that is, the ability to generate almost perfect monochromatic electromagnetic radiation) and high spatial coherence [9, 19]. In this regard, the laser beam (light) can be focused into a microscopic spot with a diameter of only slightly more than one length of the corresponding electromagnetic wave. Therefore, with a laser emitter power of only a few watts, we can obtain such an intensity of the light flux of a given electromagnetic radiation that will be a thousand times higher than the total intensity of the visible spectrum of solar radiation, averaging about $1376 \mathrm{~W} / \mathrm{m}^{2}$ at the entrance to the Earth's atmosphere $[2,9,19]$. Note that in the $1960 \mathrm{~s}$, the laser radiation intensity was about $10^{10} \mathrm{~W} / \mathrm{cm}^{2}$ [19]. In the 1970 s, it was already about $10^{15} \mathrm{~W} / \mathrm{cm}^{2}$ [19]. Until the mid-1980s, this increase in the intensity of the laser beam continued at a slow pace [19].

A. Ashkin quickly appreciated the unique capabilities of lasers almost immediately after their invention. Note that the world's first artificial ruby crystal laser containing aluminum oxide with a small admixture of chromium atoms was created in May 1960 by American physicist Theodore Harold Maiman (19272007), who worked in the research center of the Company Hughes Research Laboratories, USA [16]. As a result of a series of witty experiments conducted at Bell Labs, USA using such a laser, A. Ashkin in the 1960s invented a «light trap» that reliably holds the smallest objects of various nature [19]. These studies took him at least 25 years. In 1986, he, together with his collaborators, for the first time described in detail the experimental results obtained in the USA on optical «capture» using a «light trap» of dielectric particles ranging in size from tens of nanometers to tens of micrometers [19]. Over time, A. Ashkin's «light traps» were called «optical tweezers» or «laser tweezers». In subsequent years, this laser technology has been greatly improved and its capabilities have expanded significantly. As it turned out, «laser tweezers» can not only hold micro- and nano-objects, but can also move them, rotate and even cut them into pieces. Now they are widely used in molecular biology, genomics, virology, and in other fields of science and technology [19]. For example, laser cooling of neutral atoms to ultralow temperatures and their «trapping» has become the most important area of practical application of «optical tweezers». By the way, for his work in the field of low-temperature physics using laser technology, his former employee and US citizen Steven Chu with his compatriot William Daniel Phillips and French physicist 
Claude Cohen-Tannoudji became Nobel Laureates in physics in 1997 [2, 5].

It can be seen from the above that A. Ashkin owed his Prize to the method of manipulating nano- and microobjects using laser radiation with a relatively low energy. In contrast to A. Ashkin, his Laureate colleagues G. Mourou and D. Strickland worked in the field of laser physics with high energy indicators. By 1985, they had developed a very effective way to increase the power of laser pulses [19]. Thanks to this method, the power density of laser radiation again went into sharp growth, and now it has reached an index of up to $10^{23} \mathrm{~W} / \mathrm{cm}^{2}$ [19].

The essence of an effective method for amplifying laser pulses proposed by foreign experimental physicists G. Mourou and D. Strickland according to [19] is as follows. First, an ultrashort laser pulse of light radiation is passed through a pair of diffraction gratings, which stretch it over several orders of time. Note that in their first experiments, G. Mourou and D. Strickland used fiber optic cable for this purpose, but diffraction gratings proved to be more efficient. As a result, the peak energy of the electric field of the laser pulse drops so much that it passes through an optical radiation amplifier (a sapphire crystal doped with titanium ions is usually used for this) without disturbing (destroying) the crystal structure of the latter. Then, a repeatedly amplified laser pulse is passed through the next couple of diffraction gratings. These gratings compress it (laser pulse) to the original length (time duration). As a result, a very short laser pulse of extremely high intensity is obtained at the output of the described optical system. The first joint experiments by G. Mourou and D. Strickland, conducted by them at the Laser Energy Laboratory of the University of Rochester, USA, using this method of amplification of chirped laser pulses led to the creation of picosecond terawatt laser systems [19].

The field of practical application for today of ultrashort superpowerful laser pulses has proved to be extremely extensive, including various branches of science and technology. We point out that it extends from conducting many experiments in the field of fundamental and applied physics, utilization in military affairs (combat lasers), modifying (structuring) the surfaces of various parts, amplifying photoluminescence signals, performing hypersensitive chemical analysis of substances, creating new metamaterials, and then to surgical treatment of myopia and astigmatism in millions of people on our planet $[16,19]$.

4. Nobel Prize in physics for 2019. On October 8 , 2019, the Royal Swedish Academy of Sciences published the names of Nobel Prize winners in physics for 2019 «... for their contribution to our understanding of the evolution of the Universe and the place of the Earth in space» They are famous astronomers and astrophysicists [20]: Canadian Phillip James Edwin Peebles (born on April 25, 1935, Fig. 11) and the Swiss Michel Mayor (born on January 12, 1942, Fig. 12) and Didier Queloz (born on February 23, 1966, Fig. 13). We point out more specifically that J. Peebles was awarded this Prize «... for theoretical discoveries in physical cosmology», and M. Major and D. Queloz «... for the discovery of an exoplanet orbiting a solar-type star» [20]. In a press release from the Nobel Committee in connection with the award of this prestigious Prize, it was stated that these «... discoveries of scientists are revolutionary for astronomy» [20].

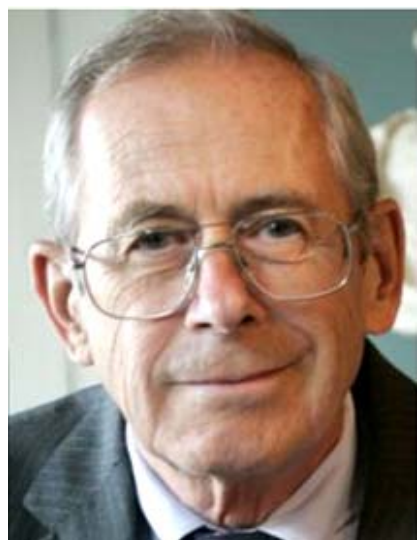

Fig. 11. Outstanding Canadian-American astrophysicist Phillip James Edwin Peebles (born in 1935), Laureate of Nobel Prize in Physics for 2019 [20]

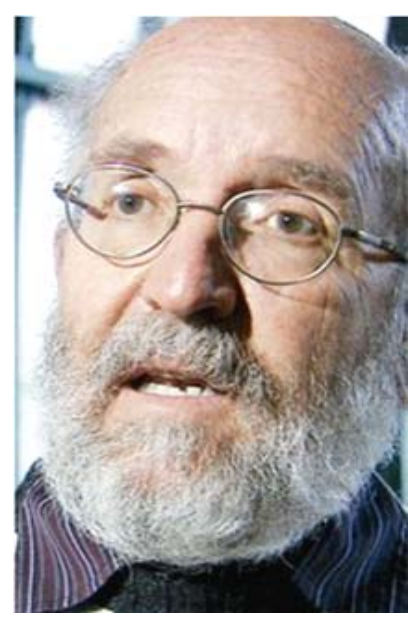

Fig. 12. Outstanding Swiss astrophysicist Michel Mayor (born in 1942), Laureate of Nobel Prize in Physics for 2019 [20]

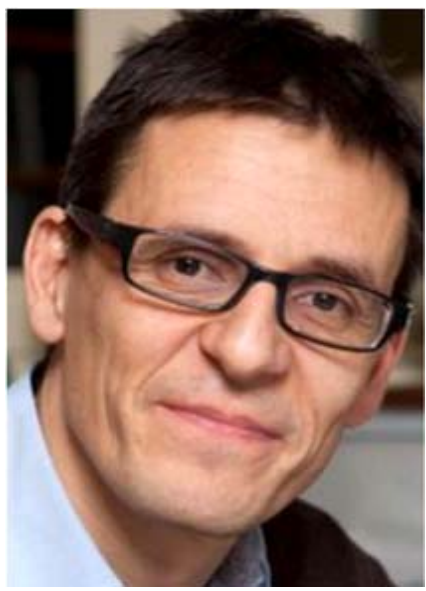

Fig. 13. Outstanding Swiss astrophysicist Didier Queloz (born in 1966), Laureate of Nobel Prize in Physics for 2019 [20] 
J. Peebles (for many years he was a Professor at Princeton University, USA) turned out to be one of those prominent astrophysicists in the world who theoretically predicted the existence and described the properties of relict radiation that filled our Universe after the Big Bang in it [20]. In 1964, J. Peebles theoretically came to the conclusion that space is penetrated by isotropic microwave radiation with temperature of the order of $10 \mathrm{~K}$ [20]. He published the results of his calculations in 1965 in the authoritative American scientific Journal «Astrophysical Journal» [20], which simultaneously published the experimental work of young US scientists Arno Penzias and Robert Woodrow Wilson, then members of Bell Labs, USA. The latter succeeded in measuring the background radio emission of the Milky Way galaxy using a six-meter horn satellite dish antenna [15]. Their receiving radio equipment tuned to a $7.35 \mathrm{~cm}$ long electromagnetic wave registered a weak but very stable signal, which was independent of the position of the antenna in the Earth's atmosphere. In 1964, these American scientists came to the conclusion that this electromagnetic signal could not have an intragalactic origin. They realized that they were dealing with a signal whose spectral composition corresponded to the emission spectrum of an absolutely black body heated to $3.5 \mathrm{~K}$ according to their then estimate [20]. This electromagnetic radiation came from all sides of the earthly firmament and was, in their opinion, completely isotropic [20]. This experimental work by A. Penzias and R.W. Wilson famous world astronomers consider a landmark achievement in 20th-century astrophysics. Therefore, it was no accident that in 1978 it brought them the Nobel Prize in physics [20, 21]. According to modern data, cosmic microwave relict radiation discovered by these talented physicists arose 380 thousand years after the Big Bang, which occurred about 12 billion years ago [2]. Outer space was then filled with plasma, consisting of protons, electrons, and helium ions, which was in thermodynamic equilibrium with «hot» electromagnetic radiation. When, due to the expansion of space, this plasma cooled to about $4000 \mathrm{~K}$, the formation of electroneutral atoms (first helium, and then hydrogen) began in it [20].

For greater clarity in the complex intergalactic problem under consideration, we note that the temperature of the relict radiation decreases inversely with the expansion of outer space. Since the linear dimensions of our Universe have increased by three orders of magnitude since the initial release of photons, the degree of its heating and, accordingly, the energy of the relict radiation have also decreased. According to current data, its temperature is $2.725 \mathrm{~K}[2,7,20]$. Therefore, its initial assessment by A. Penzias and R.W. Wilson, although was somewhat overpriced, is still surprisingly close to the truth. The intensity of microwave relict radiation reaches a peak at waves $1 \mathrm{~mm}$ long, not able to penetrate the Earth's atmosphere. Therefore, the discoverers actually recorded only its long-wave tail, and the full spectrum was determined much later using high-altitude balloons and spacecraft. However, these scientists determined the general form of this spectrum extremely accurately, although their instruments were far from perfect [20].

It should be noted that the predictions of J. Peebles are associated not with the fact of the existence of microwave relict radiation in the Universe, but with its properties [22]. Firstly, this Nobel Laureate on the basis of theoretical models developed by him showed that relict radiation plays an important role in the formation of galaxies of the Universe. Secondly, he calculated the spectrum of fluctuations of this relict radiation. He developed a new model of the Universe and with its help estimated how this radiation would look if you add in it (this model) «cold dark» matter to the ordinary matter of the Universe. Thirdly, he added «dark» energy with negative density to this model and again recalculated the spectrum of relict radiation. On a similar cosmological model, J. Peebles studied how the hypothetical entities introduced by him affect the evolution of the Universe as a whole and the formation of its galaxies in particular. Essentially, he laid the foundations of theoretical cosmology. Here, we should not forget about the great contribution to this science made by the famous Soviet theoretical physicist Yakov Borisovich Zeldovich (19141987) [16].

M. Mayor and D. Queloz, working at the Geneva Observatory, practically in 1994, jointly began a systematic search for exoplanets (earth-like planets) in the Milky Way galaxy, located near its «normal» (lying on the main astro sequence) stars and « red giants». Exoplanets previously discovered before them by other astronomers of the world, located near pulsars [15], did not present special prospects for science. Valuable to astrophysicists were only those exoplanets that were placed around stars of the solar type. In these astronomical observations, they used a unique highresolution spectrometer ELODIE (it was commissioned at the end of 1993) mounted on a 193-cm telescope of the observatory [20]. In the fall of 1994, using the indicated equipment, these scientists discovered that one of the observed stars («51 Pegasus») showed fluctuations in its radial velocity with period of about four Earth days. It was for this (for observing peculiar «star swings» from Earth) that they needed an ultraprecise spectrometer that could sense the weak shifts of the emission spectrum of stars accompanying the rotation of exoplanets around them. On November 23, 1995, M. Major and D. Queloz published their paper in the Journal Nature, from which the world learned about the long-awaited discovery of the planet orbiting an ordinary star of the main astro sequence [20]. In such a way they discovered the first exoplanet (Fig. 14) from that family of planets, which they later called the «hot» Jupiters. The newly discovered exoplanet «51 Pegasus b» was nothing like the satellites of the Sun. She circled in a circular trajectory with radius of about 7.5 million kilometers, making one revolution around her star 
in just 4.2 Earth days. At the same time, it had a very large mass - about 0.47 of the mass of Jupiter. Further in 1995, discoveries by other astronomers of two more exoplanets with mass of several Jupiters orbiting the stars «70 Virgo» and «47 Ursa Major» [20] followed. Since then, planetary astronomy once and for all went beyond the limits of the solar system. And then similar discoveries rained down one after another. We point out that after such discoveries in science, astronomers who believed in the technical capabilities of their highprecision measuring instruments have already discovered more than 4,100 exoplanets (new «worlds») scattered across our huge Milky Way galaxy [20, 22].

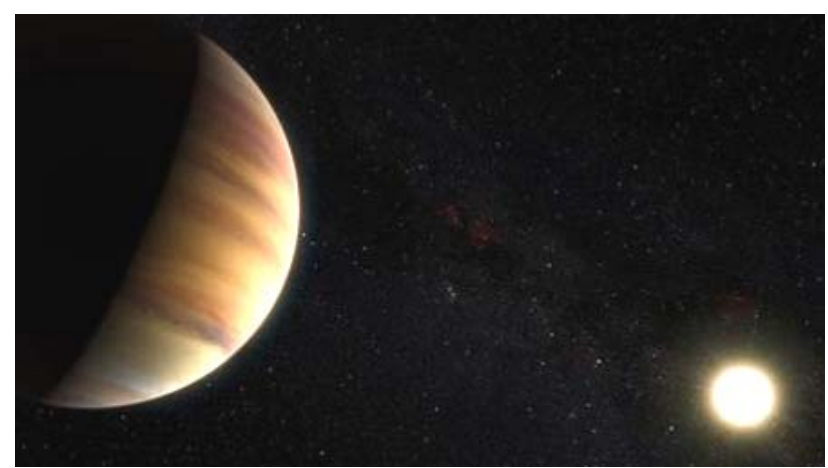

Fig. 14. General view of the exoplanet «51 Pegasus b» (left), discovered in 1995 in our Milky Way galaxy by prominent

Swiss astrophysicists M. Mayor and D. Queloz [22]

The successful «pursuit» of exoplanets not only gave astronomy rich information, but also attracted public attention to this ancient science and increased its prestige. Therefore, it is not surprising that in the 21 st century, the world scientific community made serious efforts to develop new astronomical devices of the next generations, designed for search of such exoplanets to be potentially inhabited by intelligent beings in space [20, 22]. The valuable results of astrophysical research by $\mathrm{M}$. Major and D. Queloz brought astronomy to a new path of its further development. Summarizing, we can reasonably say that J. Peebles and M. Major with D. Queloz, through their outstanding scientific works in astronomy and astrophysics, have opened up a huge wealth of new ways in studying the space surrounding us.

\section{Conclusions.}

Winners of the Nobel Prize in Physics for the period 2016-2019 made a great contribution to the further development of world science and modern technology. Their outstanding scientific and technical achievements allow to expand and deepen people's knowledge about matter, about the evolution of our Universe, to understand the place occupied by planet Earth and its role in space, and to effectively direct advanced laser technologies developed in the world to serve humanity.

\section{REFERENCES}

1. Available

https://elementy.ru/novosti nauki/432847/Nobelevskaya premi ya po_fizike_2016 (accessed 15 May 2018). (Rus).
2. Baranov M.I. Antologija vydajushhihsja dostizhenij v nauke i tehnike: Monografija v 4-h tomah. Tom 4 [An anthology of the distinguished achievements in science and technique: Monograph in 4 vols. Vol. 4]. Kharkiv, PhPB Panov A.N. Publ., 2016. 415 p. (Rus).

3. Available

https://lenta.ru/news/2017/10/03/nobelprizeinphysics (accessed 23 June 2018). (Rus).

4. Baranov M.I. An anthology of the distinguished achievements in science and technique. Part 35: Nobel Prize Laureates in Physics for 1990-1994. Electrical engineering \& electromechanics, 2016, no. 6, pp. 3-8. doi: 10.20998/2074272X.2016.6.01.

5. Baranov M.I. An anthology of the distinguished achievements in science and technique. Part 36: Nobel Prize Laureates in Physics for 1995-1999. Electrical engineering \& electromechanics, 2017, no. 1, pp. 3-9. doi: 10.20998/2074272X.2017.1.01.

6. Baranov M.I. An anthology of the distinguished achievements in science and technique. Part 37: Nobel Prize Laureates in Physics for 2000-2004. Electrical engineering \& electromechanics, 2017, no. 2, pp. 3-12. doi: 10.20998/2074272X.2017.2.01.

7. Baranov M.I. An anthology of the distinguished achievements in science and technique. Part 38: Nobel Prize Laureates in Physics for 2005-2010. Electrical engineering \& electromechanics, 2017, no. 3, pp. 3-15. doi: 10.20998/2074272X.2017.3.01.

8. Baranov M.I. An anthology of the distinguished achievements in science and technique. Part 39: Nobel Prize Laureates in Physics for 2011-2015. Electrical engineering \& electromechanics, 2017, no. 4, pp. 3-9. doi: 10.20998/2074272X.2017.4.01.

9. Kuz'michev V.E. Zakony i formuly fiziki [Laws and formulas of physics]. Kiev, Naukova Dumka Publ., 1989. 864 p. (Rus).

10. Bol'shoj illjustrirovannyj slovar' inostrannyh slov [Large illustrated dictionary of foreign words]. Moscow, Russkie slovari Publ., 2004. 957 p. (Rus).

11. Available at:

https://www.gazeta.ru/science/2016/10/04 a 10229453.shtml (accessed 15 May 2018). (Rus).

12. Available

https://www.nobelprize.org/prizes/physics/2016/summary (accessed 16 June 2018). (Rus).

13. Available

https://elementy.ru/novosti_nauki/433128/Nobelevskaya_premi ya po fizike 2017 (accessed 26 June 2018). (Rus).

14. Available

https://lenta.ru/news/2017/10/03/nobelprizeinphysics (accessed 25 April 2018). (Rus).

15. Baranov M.I. Antologiia vydaiushchikhsia dostizhenii v nauke i tekhnike: Monografiia $v$ 4-kh tomakh. Tom 2. [An anthology of outstanding achievements in science and technology: Monographs in 4 vols. Vol. 2]. Kharkov, NTMT Publ., 2013. 333 p. (Rus).

16. Baranov M.I. Antologiia vydaiushchikhsia dostizhenii v nauke i tekhnike: Monografiia $v$ 4-h tomakh. Tom 1. [An anthology of outstanding achievements in science and technology: Monographs in 4 vols. Vol. 1]. Kharkov, NTMT Publ., 2011. 311 p. (Rus).

17. Available at: https://fakty.ua/246875-obyavleny-laureatynobelevskoj-premii-po-fizike-za-2017-god (accessed 06 April 2018). (Rus). 
18. Available at: https://www.popmech.ru/science/390252nobelevskaya-premiya-po-fizike-2017-gravitacionnye-volny (accessed 16 October 2018). (Rus).

19. Available

at: https://elementy.ru/novosti nauki/433343/Nobelevskaya premi ya po fizike 2018 (accessed 20 September 2018). (Rus).

20. Available https://elementy.ru/novosti nauki/433548/Nobelevskaya premi ya po fizike 2019 (accessed 10 November 2018). (Rus).

21. Cholakov V. Nobelevskie premii. Uchenye $i$ otkrytiia [Nobel Prizes. Scientists and discovery]. Moscow, Mir Publ., 1986. $368 \mathrm{p}$.
22. Available at: https://www.bbc.com/russian/news-49960797 (accessed 11 May 2018). (Rus).

Received 27.11.2019

M.I. Baranov, Doctor of Technical Science, Professor,

Scientific-\&-Research Planning-\&-Design Institute «Molniya», National Technical University «Kharkiv Polytechnic Institute», 47, Shevchenko Str., Kharkiv, 61013, Ukraine, phone +380 577076841 ,

e-mail: baranovmi@kpi.kharkov.ua.

How to cite this article:

Baranov M.I. An anthology of the distinguished achievements in science and technique. Part 53: Nobel Prize Laureates in Physics for 2016-2019. Electrical engineering \& electromechanics, 2020, no.2, pp. 3-12. doi: 10.20998/2074272X.2020.2.01. 